\title{
Cyclicity of Neonatal Sleep Behaviors at 25 to 30 Weeks' Postconceptional Age
}

\author{
MARK S. SCHER, MARK W. JOHNSON, AND DIANE HOLDITCH-DAVIS \\ Division of Pediatric Neurology [M.S.S., M.W.J.], Department of Pediatrics, Rainbow Babies and \\ Children's Hospital, Case Western Reserve University, Cleveland, Ohio, 44106; and School of Nursing \\ [D.H.D.], University of North Carolina, Chapel Hill, North Carolina 27599
}

\begin{abstract}
Previous sleep studies of preterm neonates describe the rudimentary expression of sleep state cyclicity after $30 \mathrm{wk}$ postconceptional age (PCA), with stability over multiple cycles only after 36 wk PCA. The research objective for this study was to determine whether sleep state cyclicity was expressed in neonates of 25-30 wk PCA, using two criteria for state identification. Our neonatal sleep consortium includes a total cohort of 359 children who were healthy and medically ill neonates who were recruited from three obstetric-neonatal services and received multiple-hour EEG sleep studies. A subset of the 33 youngest preterm infants were selected to evaluate the first of serial 2- to 3-h EEG-sleep recordings to assess the presence of sleep state cyclicity. One neonatal neurophysiologist visually assigned EEG-sleep characteristics for each record. Rapid eye movement (REM) counts and EEG discontinuity were specifically chosen to
\end{abstract}

\section{ABSTRACT}

assess whether sleep cyclicity was expressed. A combined measure of REM and EEG discontinuity were used in an autocovariance analysis to assess cycling and mean cycle duration. A mean cycle duration of $68 \pm 19$ min with a range of 37-100 min was determined from the REM-EEG discontinuity state for 24 neonates. The remaining nine infants had absent or poor sleep cyclicity. Sleep state cyclicity is expressed for a majority of neonates between 25 and 30 wk PCA, reflecting an ultradian biologic rhythm during the early perinatal stage of brain development. (Pediatr Res 57: 879-882, 2005)

GA, gestational age

PCA, postconceptional age

REM, rapid eye movement
Functional brain organization can be assessed by applying analyses to EEG-sleep state architecture and continuity measures. The observation of sequentially recurring eye movement intervals alternating with no eye movement epochs was initially described in the newborn infant as early as the 1950s (1). Such a sequence suggests either the presence of a highly periodic process or, alternatively, the temporal coincidence, with variable predictability, among physiologic behaviors that sometimes define state transitions. Early investigators suggested the presence of periodicity in infant sleep measures (2,3). Sterman et al. (4) observed periodicity from early gestational ages (GAs), beginning with the basic rest-activity cycle in the fetus and the sleep cycle in infants. Globus (5) described cyclicity at all GAs, with predictable rhythmicity

Received May 20, 2004; accepted October 13, 2004.

Correspondence: Mark S. Scher, M.D., Division of Pediatric Neurology, Rainbow Babies and Children's Hospital, 11100 Euclid Avenue, M/S 6090, Cleveland, OH 44106; e-mail Mark.Scher@uhhs.com.

This study was supported in part by the following grants to M.S.S.: NS34508, NR01894, NS01110, and NS26793.

Presented in part at the American Academy of Sleep Medicine, 17th Annual Meeting, June 2003, Chicago, IL.

DOI: 10.1203/01.PDR.0000157678.84132.A8 after 36 wk postconceptional age (PCA). Other authors stressed the clinical importance of sleep periodicity, as exemplified by reports of disturbed cyclic organization of sleep in infants with CNS disorders (6) as well as asymptomatic infants at risk for sudden infant death syndrome $(7,8)$.

Specific investigations questioned the definition of sleep state cyclicity when applying alternative methods, particularly when states were considered categorical events $(5,9,10)$. On the basis of these specific methodologic approaches, sleep cyclicity did not appear until at least 6 wk of life (11), 4-6 mo postnatally $(9,12)$, or $1 \mathrm{y}$ of life $(13)$. Using the same strict method, Borghese et al. (14) demonstrated sleep cyclicity in $40 \%$ of healthy preterm infants at 36 wk PCA, with $90 \%$ cyclicity noted by 6 mo of age. These authors suggested that preterm cyclicity may be a response to ongoing stress on the CNS, whereas cyclicity at 6 mo alternatively reflects CNS competence.

Studies of sleep state cyclicity for preterm infants who are $<30$ wk GA or PCA have not been widely reported, although some authors have suggested predictable sleep state transitions in this group of preterm infants by 27-30 wk GA, using rapid eye movements (REMs) and specific EEG patterns $(15,16)$. 
Given the improving survival rates of extremely premature infants with GAs as early as $23-24$ wk (17), determination of state periodicity may help advance neurointensive care practices and predict neurodevelopmental outcome. Our neonatal sleep consortium chose to investigate the presence of sleep cyclicity in preterm neonates who were 25-30 wk PCA, combining data from one common subcortical neuronal pathway involved in state regulation (REMs) with a cerebral measure easily identified by visual analysis (EEG discontinuity).

\section{METHODS}

From a total cohort of 359 neonates, a subset of the youngest 33 preterm infants who were 25-30 wk PCA (23-29 wk GA, 4-50 d of age) were chosen to analyze the first of serial 2- to 3-h EEG-sleep recordings. Institutional Review Board-approved informed consent was obtained for all study subjects. Our total cohort was composed of both healthy and medically ill neonates, recruited from the neonatal services of three obstetric/neonatal centers in Pittsburgh, Cleveland, and North Carolina. Medical health status was tabulated by numeric scoring methods designed for medically ill neonates (18). Clinical and demographic information identified the medical status of each child. One trained neonatal electroencephalographer/polysomnographer scored EEGsleep states using Insight (universal EEG reading software; Persyst Development Corp., Prescott, AZ), including scoring of discontinuous EEG, arousals, movements, and REM counts over a 2- or 3-h recording time. These scores were transferred to a relational database (Microsoft, Redmond, WA) to facilitate storage and processing of raw scores into epoch-by-epoch scores. Direct annotation by the EEG technician and from review of video recordings were also included in the database.

Two criteria of state identification-1) REM, a noncerebral measure represented a subcortical pathway involved in state expression, and 2) intervals of low-amplitude EEG activities, termed EEG discontinuity-represented a cerebral measure involved in corticothalamic neuronal pathways (19). Other scoring rules included arousal identification as a sudden desynchronization or diminution of amplitude of frequencies on EEG channels with coincident abrupt alteration in the respiratory rate regularity and sometimes increased electromyogram activity. Indeterminate or transitional state segments were also identified.

EEG-sleep studies were carried out in an environmentally controlled setting in which sound, light, humidity, and tactile stimulation were monitored. All infants were studied while sleeping prone or on their side in an open bed or isolette, whichever was their usual sleeping position in the nursery. Continuous recordings began after a diaper change and feeding during the late morning to early afternoon hours. Each 24-channel 2- to 3-h study was recorded using a digital EEG machine (Computational Diagnostics, Pittsburgh, PA; Nicolet Biomedical, Madison, WI; Nihon Kohden America, Foothill Ranch, CA).

A neonatal nurse assisted the EEG technologist with clinical care for each infant during the recording session. Feeding behavior, diaper changes, medication administration, and technical comments were documented in the digital EEG. No infants were given medications during the studies.

EEG-sleep studies were visually analyzed by a single neonatal neurophysiologist (M.S.S.) who specifically identified REM epochs and periods of continuous and discontinuous EEG intervals. For EEG discontinuity, the start and the end of a duration of EEG attenuation or quiescence were marked (Fig. $1 A)$.

Our analytic method applied an autocovariance analysis (see Appendix) to a measure of state. All calculations and analyses were performed using Igor Pro (Wavemetrics, Lake Oswego, OR). The state measure was defined from a weighted sum of REM per minute (upward) and seconds of EEG discontinuity per minute (downward), with weightings of +1 for REM and $-1 / 2$ for discontinuity). During epochs in which no REM and EEG discontinuity were present, such as may occur during segments of transitional or indeterminate sleep, the state measure was simply 0 . The autocovariance is a measure of similarity of a signal to itself when the signal is shifted by the time $\tau$ relative to a copy of itself. Application of the autocovariance to the state measure results in a function that is positive when the signal and its shifted copy are in phase and negative when they are out of phase. The function is bounded between -1 and +1 and cycles above and below 0 when the signal is periodic. The first dominant peak along the time shift axis (excluding $\tau=0$ ) is the dominant average cycle period of the signal. Figure $1 A$ shows a 30 -s sample of discontinuous EEG data taken from a 30 -wk preterm infant. Figure $1 B$ shows a sample of continuous EEG with REM taken from the same infant $\sim 6 \mathrm{~min}$ later. Figure 2 illustrates an example of a 27 -wk preterm infant showing the

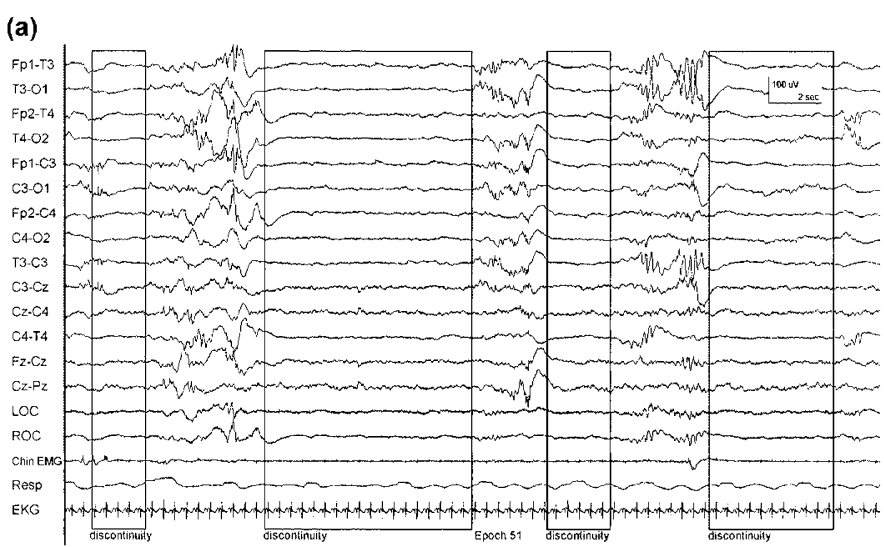

(b)

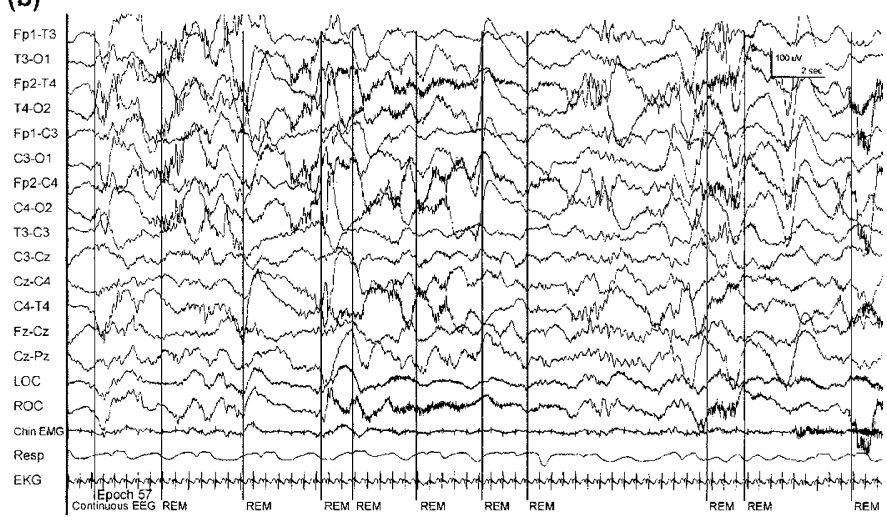

Figure 1. Segments of EEG from a preterm neonate at $30 \mathrm{wk}$ PCA. (A) Quiet sleep as indicated by the marked segments of discontinuity. (B) Active sleep as indicated by the "Continuous EEG" annotation and the occurrence of REMs. The EEG channel display settings were $30 \mathrm{~s}$ per page, $10 \mu \mathrm{V} / \mathrm{mm}$ equivalent sensitivity, neonatal montage, $0.3 \mathrm{~s}$ time constant, $35 \mathrm{~Hz}$ high filter, and $60 \mathrm{~Hz}$ notch filter on. C, central; T, temporal; P, parietal; F, frontal; O, occipital; and odd numbers left, even numbers right, and $\mathrm{z}$ along the centerline; ROC, right outer canthus; LOC, left outer canthus.

recording of REM, EEG discontinuity, and combined state epochs, followed by a smoothed graph of the autocovariance function of the combined REM/EEG state measure.

\section{RESULTS}

Twenty-four neonates showed a mean cycle duration of 68 \pm 19 min with a range of 37-100 min. Nine neonates had poor or no cyclicity present. No differences in sex, race, GA, PCA, ventilatory care, medication (e.g. theophylline), postnatal illnesses, or prenatal risk were seen between groups with recognizable cyclicity and those with poor or no cyclicity. Sleep state cyclicity was similar in duration within individuals, but cycle lengths were variable between individuals. Figures 3 and 4 indicate the combined state and autocovariance for selected preterm infants with and without recognizable cycling.

\section{DISCUSSION}

Although it is generally assumed that CNS control of sleep begins during early life (1), on the basis of the recognition of REM and non-REM cycles, Goldie (20) argued that cyclicity may not be highly predictable and may only alternatively consist of a "sequence of coincident or coalescent behaviors" earlier in life. Cyclicity was initially suggested on the basis of 

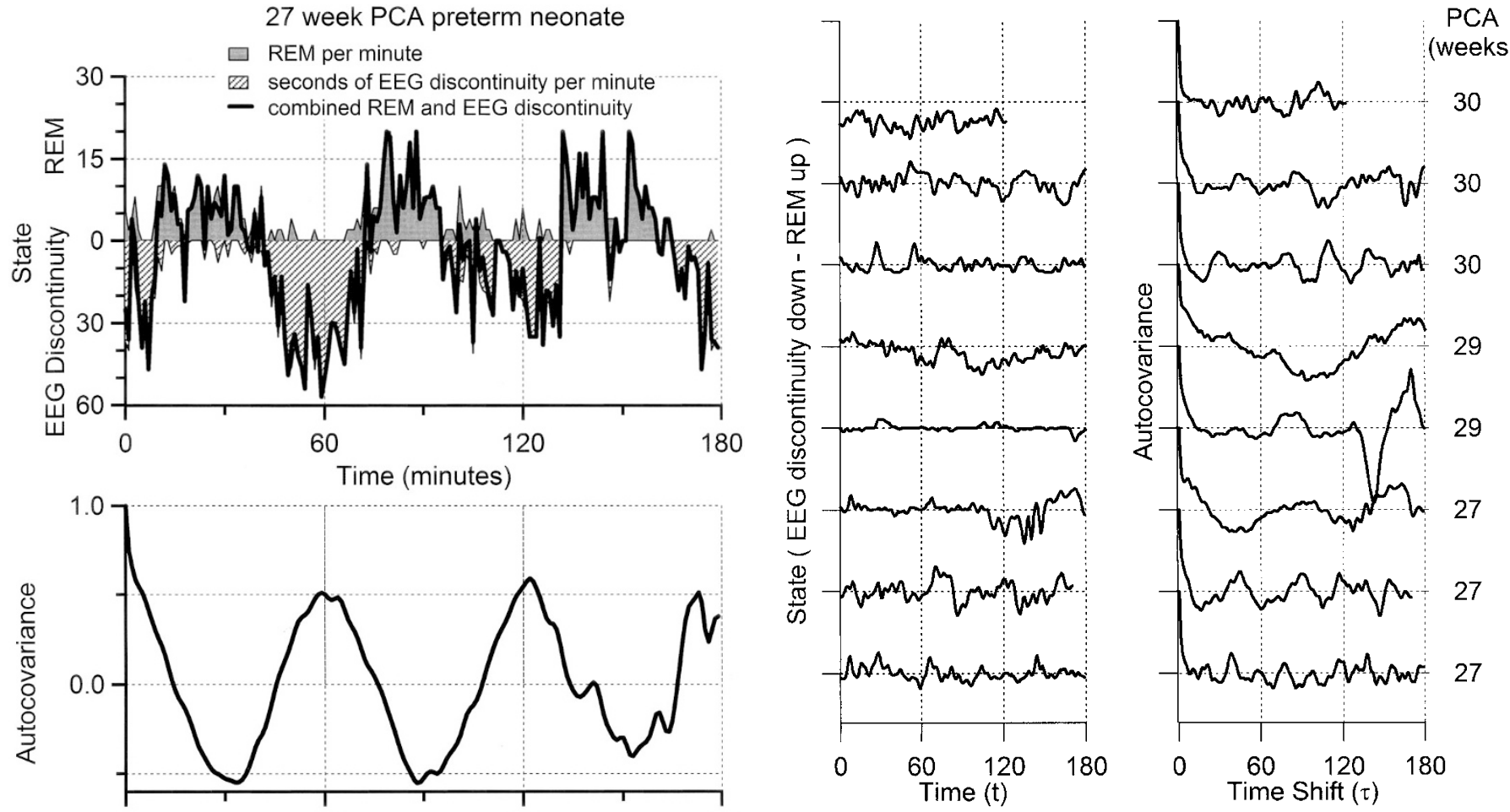

Figure 4. Selected infants who exhibited absent or poor sleep cyclicity (displayed as in Fig. 3).

Figure 2. A sample EEG-sleep cycle from a 27-wk corrected age preterm neonate. This figure illustrates the alternating occurrence of EEG discontinuity and REM based on visual analysis scoring (top) and the autocovariance function for the combined EEG state and REM (bottom).
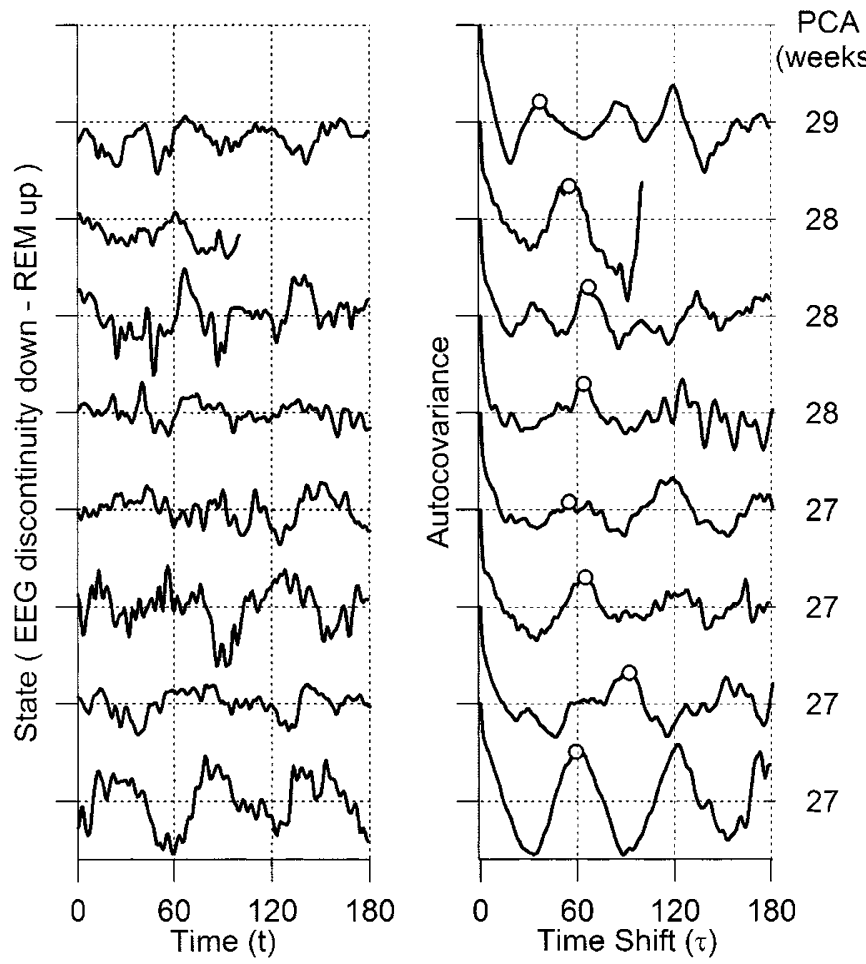

Figure 3. Selected infants who demonstrated state cyclicity. (Left) Combined state (REM and EEG discontinuity). (Right) Autocovariance function of the combined state for the same infants, with the mean for cycle length for each neonate designated by an open circle. The postconceptional age is shown to the right of each infant.

the results of studies during the neonatal period by several authors during the 1970s and early 1980s $(2,3,5,20,21)$, with the absence of cyclicity observed in encephalopathic infants (6) as well as asymptomatic high-risk infants for sudden infant death syndrome (22). Later reports by certain authors argued that more strict methods to define cyclicity failed to document sleep cycles until later during the first year of life. The appearance of sleep cyclicity was noted at $6 \mathrm{wk}$ of age (11), 4-6 mo of age (9), or 1 y of age (13).

A study using a movement-sensitive mattress to detect staterelated movements and respiratory behaviors documented cyclicity for 20 of 49 infants using these 24-h studies by $36 \mathrm{wk}$ GA (14). Thirty-seven of 42 infants of this same cohort expressed sleep cyclicity using the same method at 6 mo of age. The authors documented a similar sleep cycle duration of 60 min that remained unchanged at the two ages when studies were performed. These authors also reported the presence of cyclicity with developmental outcome using a motor-weighted developmental score (the Bayley Score of Infant Development). The presence of cyclicity during the preterm period (at 36 wk gestation) correlated negatively with development at 6 mo of age, whereas sleep cyclicity correlated positively with developmental outcome at 6 mo of age. The authors speculated that the presence of cyclicity in the very immature brain may reflect stress on functional brain organization and maturation that later negatively affects outcome.

No studies have indicated the presence or absence of sleep state cyclicity in preterm infants who are $<30$ wk GA, although selected studies have suggested the coincidence among different physiologic behaviors during state transitions $(15,16)$. Our findings suggest that preterm neonates with PCAs of 
25-30 wk do express sleep state cyclicity, based on two criteria of state identification. We chose two separate neuronal networks that represent either a subcortical circuitry involving largely the upper brainstem (REM) and cerebral circuit involving thalamocortical pathways (EEG discontinuity).

We understand that there are several limitations to the present study. First, studies were performed over $2-3 \mathrm{~h}$ between feedings, which were too short to ascertain the diurnal effect on sleep state cyclicity, as well as the stability of sleep state cycling over a 24-h period. Second, electrographic detection of REMs using electro-oculographic channels (without supplementation by visual observation) would not be optimal during the preterm ages for all subjects. During this range of prematurity, eye movements create only a weak dipole because of an immature retina, sometimes resulting in a lack of discernible deflections on the electro-oculogram channels. Third, the number of days spent in the nursery and the child's sleep position compared with the other infants studied might influence cyclicity. These three limitations may in part explain why poor or absent sleep state cyclicity was noted for nine of our infants. Finally, our present population size is too small to compare neurodevelopmental outcome in those with and without cyclicity. Despite these limitations, we conclude that the presence of sleep state cyclicity exists for a majority of infants who are $<30$ wk PCA, based on an autocovariance estimate of sleep state cyclicity, independent of demographic or selected clinical influences.

\section{APPENDIX}

The "correlation" of two signals is a measure of their similarity as a function of a time shift, $\tau$. The correlation of any two signals $\mathrm{X}$ and $\mathrm{Y}$ is defined by

Correl $_{\mathrm{XY}}[\tau]=\Sigma \times[\tau] \mathrm{Y}[\mathrm{t}+\tau]$

where $\tau$ is the time shift between the signals, $\mathrm{t}$ is time, and the function is summed over all valid values of $t$. The "covariance" is defined using the same equation after subtracting the mean value of $\mathrm{X}$ from $\mathrm{X}$ and the mean value of $\mathrm{Y}$ from $\mathrm{Y}$. "Autocovariance" is the covariance of any signal with itself. Autocovariance presented here uses linear correlation, which adds a sequence of zeros to the ends of the signal, rather than circular correlation, which wraps the signal back on itself. Finally, the autocovariance is normalized by the value at $\tau=$ 0 and corrected for the number of minutes used at each value of $\tau$, resulting in a function that is exactly equal to 1 at $\tau=0$, which is generally bounded between -1 and +1 and cycles above and below 0 when the signal is periodic, with the first dominant peak along the time shift axis (excluding $\tau=0$ ) being the dominant period of the signal.

Acknowledgments Dawn Salerno, Marquita Beggarly, Judy Gutman, Jennifer Avery, and Michael Harrison performed the EEG-sleep studies.

\section{REFERENCES}

1. Aserinsky E, Kleitman N 1955 A motility cycle in sleeping infants as manifested by ocular and gross bodily activity. J Appl Physiol 8:11-18

2. Theorell K, Prechtl HF, Blair AW, Lind J 1973 Behavioural state cycles of normal newborn infants. A comparison of the effects of early and late cord clamping. Dev Med Child Neurol 15:597-605

3. Stern E, Parmelee AH, Harris MA 1973 Sleep state periodicity in prematures and young infants. Dev Psychobiol 6:357-365

4. Sterman MB, Harper RM, Havens B, Hoppenbrouwers T, McGinty DJ, Hodgman JE 1977 Quantitative analysis of infant EEG development during quiet sleep. Electroencephalogr Clin Neurophysiol 43:371-385

5. Globus GG 1970 Quantification of the REM sleep cycle as a rhythm. Psychophysiology 7:248-253

6. Dreyfus-Brisac C 1970 Ontogenesis of sleep in human prematures after 32 weeks of conceptional age. Dev Psychobiol 3:91-121

7. Harper RM, Leake B, Hoffman H, Walter DO, Hoppenbrouwers T, Hodgman J, Sterman MB 1981 Periodicity of sleep states is altered in infants at risk for the sudden infant death syndrome. Science 213:1030-1032

8. Navelet Y, Payan C, Guilhaume A, Benoit O 1984 Nocturnal sleep organization in infants "at risk" for sudden infant death syndrome. Pediatr Res 18:654-657

9. Harper RM 1983 Infant sleep development. In: Mayes A (ed) Sleep Mechanisms and Functions. United Kingdom, Van Nostrand Reinhold Co Ltd, pp 107-125

10. Kraemer HC, Hole WT, Anders TF 1984 The detection of behavioral state cycles and classification of temporal structure in behavioral states. Sleep 7:3-17

11. Coons S, Guilleminault C 1984 Development of consolidated sleep and wakeful periods in relation to the day/night cycle in infancy. Dev Med Child Neurol 26:169-176

12. Harper RM, Leake B, Miyahara L, Hoppenbrouwers T, Sterman MB, Hodgman J 1981 Development of ultradian periodicity and coalescence at 1 cycle per hour in electroencephalographic activity. Exp Neurol 73:127-143

13. Anders TF, Keener M 1985 Developmental course of nighttime sleep-wake patterns in full-term and premature infants during the first year of life. I. Sleep 8:173-192

14. Borghese IF, Minard KL, Thoman EB 1995 Sleep rhythmicity in premature infants: implications for development status. Sleep 18:523-530

15. Curzi-Dascalova L, Figueroa JM, Eiselt M, Christova E, Virassamy A, d'Allest AM, Guimaraes H, Gaultier C, Dehan M 1993 Sleep state organization in premature infants of less than 35 weeks' gestational age. Pediatr Res 34:624-628

16. Curzi-Dascalova L 2001 Between-sleep states transitions in premature babies. J Sleep Res 10:153-158

17. Bhutta AT, Cleves MA, Casey PH, Cradock MM, Anand KJ 2002 Cognitive and behavioral outcomes of school-aged children who were born preterm: a metaanalysis. JAMA 288:728-737

18. Brazy JE, Goldstein RF, Oehler JM, Gustafson KE, Thompson RJ Jr 1993 Nursery neurobiologic risk score: levels of risk and relationships with nonmedical factors. J Dev Behav Pediatr 14:375-380

19. España RA, Scammell TE 2004 Sleep neurobiology for the clinician. Sleep 27:811820

20. Goldie L 1965 Sleep cycles in premature infants. Dev Med Child Neurol 47:317-319

21. Parmelee AH 1974 Ontogeny of sleep patterns and associated periodicities in infants. Mod Probl Paediatr 13:298-311

22. Harper RM, Frostig Z, Taube D, Hoppenbrouwers T, Hodgman JE 1983 Development of sleep-waking temporal sequencing in infants at risk for the sudden infant death syndrome. Exp Neurol 79:821-829 